\title{
Gravito-magnetic instabilities in anisotropically expanding fluids
}

\author{
Kostas Kleidis ${ }^{1,2}$, Apostolos Kuiroukidis ${ }^{1,3}$, Demetrios B Papadopoulos ${ }^{1}$ and Loukas Vlahos ${ }^{1}$ \\ ${ }^{1}$ Department of Physics, Aristotle University of Thessaloniki, 54124 Thessaloniki, Greece \\ ${ }^{2}$ Department of Civil Engineering, Technological Education Institute of Serres, 62124 Serres, Greece and \\ ${ }^{3}$ Department of Informatics, Technological Education Institute of Serres, 62124 Serres, Greece
}

(Dated: June 16, 2018)

\begin{abstract}
Gravitational instabilities in a magnetized Friedman - Robertson - Walker (FRW) Universe, in which the magnetic field was assumed to be too weak to destroy the isotropy of the model, are known and have been studied in the past. Accordingly, it became evident that the external magnetic field disfavors the perturbations' growth, suppressing the corresponding rate by an amount proportional to its strength. However, the spatial isotropy of the FRW Universe is not compatible with the presence of large-scale magnetic fields. Therefore, in this article we use the general-relativistic (GR) version of the (linearized) perturbed magnetohydrodynamic equations with and without resistivity, to discuss a generalized Jeans criterion and the potential formation of density condensations within a class of homogeneous and anisotropically expanding, self-gravitating, magnetized fluids in curved space-time. We find that, for a wide variety of anisotropic cosmological models, gravito-magnetic instabilities can lead to sub-horizonal, magnetized condensations. In the non-resistive case, the power spectrum of the unstable cosmological perturbations suggests that most of the power is concentrated on large scales ( small $k$ ), very close to the horizon. On the other hand, in a resistive medium, the critical wave-numbers so obtained, exhibit a delicate dependence on resistivity, resulting in the reduction of the corresponding Jeans lengths to smaller scales (well bellow the horizon) than the non-resistive ones, while increasing the range of cosmological models which admit such an instability.
\end{abstract}

PACS numbers: 04.25.Nx, 04.40.Nr, 04.20.Jb

\section{INTRODUCTION}

It is known that, the formation of large-scale structures (such as galaxies, super-clusters of galaxies and/or superclouds of stars within galaxies) both in cosmological and in astrophysical scale is closely related to the concept of instability [1], [2].

The first attempt towards a theory of galaxy formation carried out by Sir James Jeans in the early nineteenths [3], [4]. He assumed that the Universe is filled with a self-gravitating non-relativistic fluid, characterized by its mass-density $(\rho)$, the corresponding pressure $(p)$ and a velocity field $(\vec{u})$. As a consequence, the resulting dynamical system is governed by the equation of continuity, together with Euler's and Newton's equations for the matter-content and the gravitational field, respectively [5]. However, Jeans' theory does not apply to the formation of galaxies in an expanding Universe, because it involves a static medium. Nevertheless, he arrived at the conclusion that, density fluctuations with wavelengths $\lambda$ greater than a critical value $\lambda_{J}$ will grow, so that the system, eventually, becomes unstable (gravitational instability). As a consequence, any isothermal gaseous sphere with a length-scale greater than $\lambda_{J}$ is gravitationally unstable and is going to contract [6].

The first satisfactory theory of gravitational instabilities in an expanding Universe was proposed by Lifshitz [7]. He showed that, in a homogeneous and isotropic Universe filled with a perfect fluid, cosmological perturbations with wave-numbers $(k)$ below the Jeans' one $\left(k_{J}\right)$ grow, not exponentially, but, as a power of the corresponding scale factor, $R(t)$. Among the various scenarios of cosmic fluctuations there is also the case where gravitation and cosmic expansion are essentially irrelevant. In this context, Weinberg's work [8] is of particular interest: For the first time, the notion of an imperfect fluid was used and the role of dissipation in the survival of protogalaxies was taken into account, along the lines of Eckart's formalism [9], which differs from the corresponding of Landau \& Lifshitz [10]. Accordingly, Weinberg found that the protogalactic fluctuations, which behave as ordinary sound waves during the period where their matter-content is much lower than the corresponding Jeans mass $\left(M<<M_{J}\right)$, damp in the acoustic phase due to the viscosity of the imperfect fluid.

The study of gravitational instabilities in the presence of magnetic fields in an expanding Universe arose as a natural generalization [11]. Accordingly, solutions for the evolution of the mass-density and the magnetic field $\left(H^{i}\right)$ perturbations (in the linear regime) have been quested in FRW cosmological models, in which, the magnetic field was assumed to be too weak to destroy the isotropy of the model [12] - [16].

Although it has been argued that a uniform magnetic field slows down the growth-rate of the unstable modes, several authors have studied the influence of cosmological magnetic fields (either homogeneous or inhomogeneous) to the formation of structures within a cosmic medium (e.g. see [17] - [25], for an extensive, though incomplete list). In this context, it was shown [26] that several magnetohydrodynamic processes like pinch-effects, hoseinstabilities, sausage- and kink-instabilities, may also play a significant role to structure formation. These processes correspond to individual harmonics in the eigenfunction solutions of the wave equation for plasma instabilities and although they are different, their time- 
development is similar.

In the present article, we intend to discuss gravitational instabilities in a homogeneous and anisotropic cosmological model, in which the large-scale anisotropy is due to the presence of a homogeneous magnetic field along the $\hat{z}$-direction.

Our choice is justified by the fact that, mathematically speaking, the spatial isotropy of the FRW Universe is not compatible with the presence of large-scale magnetic fields. In fact, an anisotropic cosmological model can and should be imposed for the treatment of magnetic fields whose coherent length is comparable to the horizon length [27]. Therefore, although current observations give a strong motivation for the adoption of a FRW model, the effects one may lose by neglecting the largescale anisotropy induced by the background magnetic field, should be investigated. Not to mention that the anisotropy of the unperturbed model facilitates a closer study of the coupling between magnetism and geometry.

The Paper is organized as follows: In Section II, we summarize the general-relativistic version of magnetohydrodynamics (MHD) and present its application to the Thorne's class of homogeneous and anisotropic cosmological models [28]. They correspond to a class of semirealistic solutions to the Einstein-Maxwell equations, adequate to describe a model which departs from isotropy along the radiation epoch [29]. In Section III, we perturb the corresponding MHD equations to study the evolution of linear fluctuations propagating along the $\hat{x}$-direction, i.e. perpendicularly to the magnetic field. An immediate result is the derivation of the corresponding dispersion relation, which may reveal the critical values of the perturbations' wave-numbers in order to discuss on their potential growth (or decay). We find that, for a wide class of anisotropic cosmological models, gravito-magnetic instabilities can lead to sub-horizonal, magnetized condensations. In this case, the power spectrum of the unstable cosmological perturbations suggests that most of the power is concentrated on large scales (small $k$ ), close to the horizon. These results are in contrast to what has been found in the isotropic case, where the magnetic field disfavors large-scale matter aggregation, by reducing the growth-rate of the corresponding density perturbations [11], [13], [14]. In Section IV, we consider the relativistic MHD equations within a resistive medium. Now, the critical wave-numbers so obtained exhibit a delicate dependence on resistivity, resulting in the reduction of the corresponding Jeans lengths to smaller scales than the non-resistive ones, while increasing the range of cosmological models which admit such an instability.

\section{MAGNETOHYDRODYNAMICS IN A MODEL UNIVERSE}

In the system of geometrical units, where both the velocity of light $(c)$ and Newton's constant $(G)$ are equal to unity, the GR version of the MHD-equations [18] reads

$$
\begin{aligned}
& \left(\rho-\frac{H^{2}}{2}\right)_{; \alpha \beta} u^{\alpha} u^{\beta}=h^{\alpha \beta}\left(p+\frac{H^{2}}{2}\right)_{; \alpha \beta}+2 \frac{d}{d t}\left(H^{2} \theta\right) \\
& -\left(H^{\alpha} H^{\beta}\right)_{; \alpha \beta}+2 \chi\left(\frac{2 \theta^{2}}{3}+\sigma^{2}-\omega^{2}-\dot{u}^{\alpha} \dot{u}_{\alpha}\right) \\
& +\frac{\chi}{2}\left(\rho+3 p+H^{2}\right)+2 \dot{u}_{\alpha}\left(H^{\alpha} H^{\beta}\right)_{; \beta}+\left(H^{2}\right)_{; \alpha} \dot{u}^{\alpha}
\end{aligned}
$$

supplemented by the equation of motion $T_{; \beta}^{\alpha \beta}=0$

$$
\dot{\chi} u^{\alpha}+\chi \dot{u}^{\alpha}+\chi \theta u^{\alpha}+\left(p+\frac{H^{2}}{2}\right)_{; \beta} g^{\alpha \beta}-\left(H^{\alpha} H^{\beta}\right)_{; \beta}=0
$$

and Maxwell's equations in curved space-time

$$
\dot{H}^{\mu}=\left(\sigma_{\nu}^{\mu}+\omega_{\nu}^{\mu}-\frac{2}{3} \delta_{\nu}^{\mu} \theta\right) H^{\nu}+\frac{1}{\rho+p} p_{, \nu} H^{\nu} u^{\mu}
$$

In Eqs (1) - (3), the dot denotes differentiation with respect to $t$, Greek indices refer to the four-dimensional space-time (in accordance, Latin indices refer to the three-dimensional spatial section) and $\delta_{\nu}^{\mu}$ is the Kronecker symbol. Furthermore, $\rho$ is the mass-energy density as measured in the rest-frame of the fluid, $p$ is the corresponding pressure and $H^{\mu}$ are the components of the magnetic field. Accordingly, $\chi$ stands for the combination $\chi=\rho+p+H^{2}, u^{\alpha}$ is the fluid's four-velocity and $h^{\alpha \beta}=g^{\alpha \beta}+u^{\alpha} u^{\beta}$ is the projection tensor. Finally, $\sigma_{\mu \nu}$ and $\omega_{\mu \nu}$ stand for the shear- and the rotation- tensor respectively, while $\theta=u_{; \alpha}^{\alpha}$ is the expansion parameter, with the semicolon denoting covariant derivative. Accordingly, $\dot{u}^{\alpha}=u_{; \beta}^{\alpha} u^{\beta}$. The above system is supplemented by the Raychaudhuri equation [30]

$$
\dot{u}_{; \alpha}^{\alpha}=\dot{\theta}+\frac{\theta^{2}}{3}+2\left(\sigma^{2}-\omega^{2}\right)+\frac{1}{2}\left(\rho+3 p+H^{2}\right)
$$

Eqs (1) - (4) govern the evolution of magnetized plasmafluids within the context of GR. In what follows, we use them to describe the propagation of small-amplitude waves (cosmological perturbations) in a homogeneous and anisotropic cosmological model, the anisotropy of which is due to the presence of an ambient magnetic field (frozen into the matter-content) along the $\hat{z}$-direction, $\vec{H}=H(t) \hat{z}$.

The corresponding line-element is written in the form [28], [29], [31]

$$
d s^{2}=-d t^{2}+A^{2}(t)\left(d x^{2}+d y^{2}\right)+W^{2}(t) d z^{2}
$$

In Eq (5), the scale factors $A(t)$ and $W(t)$ are solutions to the Einstein-Maxwell equations for a magnetized perfectfluid obeying the equation of state

$$
p=\gamma \rho
$$

Therefore, the various models of this class are parameterized by $\gamma$ taking values in the interval $\gamma \in\left[\frac{1}{3}, 1\right)$. 
Accordingly, $A(t) \sim t^{1 / 2}$ and $W(t) \sim t^{l}$, where $l=$ $(1-\gamma) /(1+\gamma)[28]$, while

$$
\rho(\gamma, t)=\frac{3-\gamma}{2 t^{2}(1+\gamma)^{2}}
$$

and

$$
H(\gamma, t)=\frac{(1-\gamma)^{1 / 2}(3 \gamma-1)^{1 / 2}}{2 t(1+\gamma)}
$$

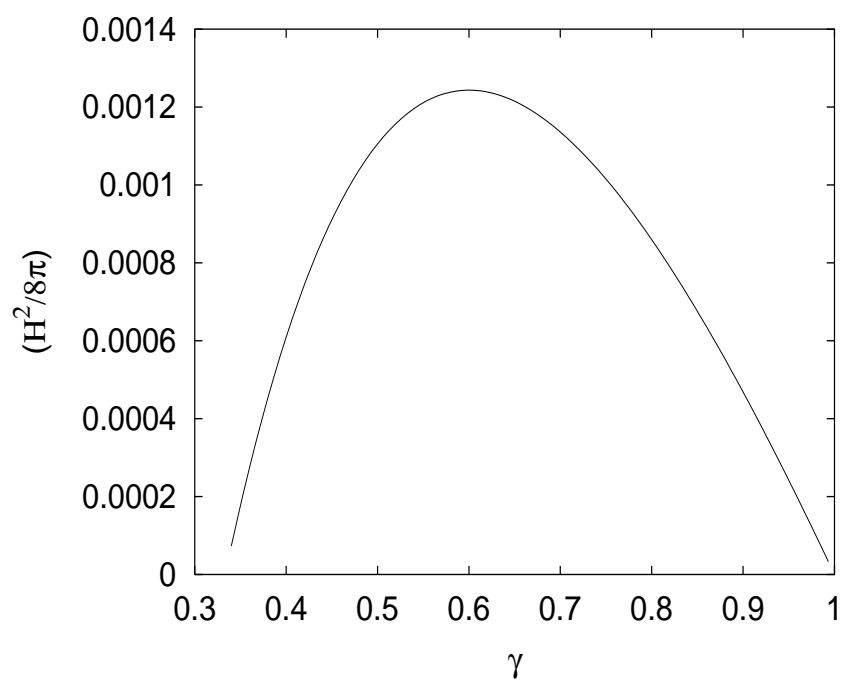

FIG. 1: The energy-density of the magnetic field $\left(H^{2} / 8 \pi\right)$ in cgs units, at a fixed time $\left(t \simeq 10^{-21} \mathrm{sec}\right)$, as a function of $\gamma$ taking values in the range $\left(\frac{1}{3}, 1\right)$. It becomes maximum in a cosmological model with $\gamma=0.6$.

Notice, that for $\gamma=\frac{1}{3}$ the strength of the magnetic field vanishes and the model reduces to the radiationdominated FRW model. As $t \rightarrow 0$, these models evolve towards a singularity of infinite density and magnetic field. However, for every $\frac{1}{3}<\gamma<1$, the ratio

$$
\frac{\rho_{\text {magn }}}{\rho_{\text {matt }}}=\frac{H^{2}}{8 \pi \rho}=\frac{(1-\gamma)(3 \gamma-1)}{16 \pi(3-\gamma)}
$$

remains (a non-zero) constant, suggesting that the influence of the magnetic field to the evolution of the curved space-time is comparable to that of the matter.

We assume (e.g. see [29]) that the metric (5) can describe the early Universe prior to the recombination epoch $\left(t_{\text {rec }} \sim 10^{13} \mathrm{sec}\right)$. At $t=t_{\text {rec }}$ (i.e. far away from the initial singularity) the magnetic field is too weak to have any effect on the dynamics of the model, but preserves the inherent anisotropy. Therefore, the spatial section of (5) always remain axially-symmetric. In fact, it is the magnetic field that accelerates the transverse expansion (over the $x y$-plane), while decelerating the longitudinal one along the $\hat{z}$-direction [28].

For an observer comoving with the fluid in the spacetime (5), we obtain

$$
\dot{u}^{\mu}=0, \quad \omega_{\mu \nu}=0, \quad \theta=\frac{2}{(1+\gamma) t}
$$

and

$$
\sigma^{2}=\frac{(3 \gamma-1)^{2}}{6(1+\gamma)^{2} t^{2}}
$$

To study the conditions under which the background (5) admits gravitational instabilities or not, we turn to the theory of cosmological perturbations.

\section{JEANS LENGTHS IN A MAGNETIZED PERFECT FLUID}

For every dynamical system, much can be learnt by investigating the possible modes of small-amplitude oscillations or waves. A plasma is physically much more complicated than an ideal gas, especially when there is an externally applied magnetic field. As a result, a variety of small-scale perturbations may appear.

Accordingly, we perturb Eqs (1) - (4), introducing (to the physical variables of the fluid) small-amplitude fluctuations along the $\hat{x}$-axis and neglect all terms of orders higher or equal than the second. Within the limits of linear analysis, excitation of cosmological perturbations in a homogeneous and anisotropic cosmological model is basically a kinematic effect, in the sense that the self-gravitation of the fluctuations is unimportant (e.g. see [6], pp. 501 - 506 and references therein). In this case, perturbations' growth arises mostly due to their motion in the anisotropic background. Therefore, as far as the enhancement of MHD perturbations in the anisotropic space-time (5) is concerned, we may neglect the first-order corrections of the metric, admitting the so-called Cowling approximation [32]. In other words, in what follows, we treat the MHD perturbations as very slowly-varying (low) frequency waves propagating in an anisotropically expanding medium, without interacting with the curved space-time, unless the linear regime breaks down.

To develop the theory of small-amplitude waves in the curved space-time (5), we follow the so-called adiabatic approximation [1], [34], [35] considering that all the perturbation quantities are proportional to the exponential

$$
\exp \left[\imath\left(k x-\int^{t} n\left(t^{\prime}\right) d t^{\prime}\right)\right]
$$

where, $k$ is the comoving wave-number. In this context, the (slowly-varying) time-dependent frequency of the wave $[n(t)]$ is defined by the eikonal

$$
\Omega=\int^{t} n\left(t^{\prime}\right) d t^{\prime}
$$

through the relation $n=d \Omega / d t$. Accordingly, we assume a wave-like expansion of the perturbation quantities, in the form

$$
\begin{aligned}
\delta \rho=A_{\rho} e^{\imath\left(k x-\int^{t} n d t^{\prime}\right)}, & \delta u=A_{u} e^{\imath\left(k x-\int^{t} n d t^{\prime}\right)} \\
\delta H & =A_{H} e^{\imath\left(k x-\int^{t} n d t^{\prime}\right)}, \quad \delta p=c_{s}^{2} \delta \rho
\end{aligned}
$$


where $c_{s}^{2}=\delta p / \delta \rho=\gamma$ is the speed of sound (in units of $c$ ). In the anisotropic model under consideration, the MHD equations (1) - (4) linearized over the perturbation quantities, result in

$$
\delta u\left[2 \chi \Gamma_{01}^{1}-g^{33} \Gamma_{33}^{0} H^{2}+p_{, 0}^{*}+\imath n \chi\right]=\imath k[\gamma \delta \rho+H \delta H]
$$

and

$$
(H \delta H)[(2-\gamma) \theta+\imath n]=\frac{u_{A}^{2}}{1+\gamma}[\theta(1+\gamma)-\imath n] \delta \rho
$$

where $\Gamma_{\alpha \beta}^{\gamma}$ are the Christoffel symbols of (5), $g^{\mu \nu}$ are the contravariant components of the corresponding metric tensor, $u_{A}^{2}=\frac{H^{2}}{\rho}$ is the (dimensionless) Alfvén velocity [clf Eq (9)] and we have set $p^{*}=p+\frac{H^{2}}{2}$.

Before attempting to discuss any temporal evolution of the perturbation quantities, it is important to trace what kind of waveforms are admitted by this system. To do so, we have to derive their dispersion relation, $D(k, n)=0$ [35]. Provided that certain kinds of modes (such as acoustic, magnetosonic etc) do exist, they can be excited through their interaction with the anisotropic space-time. An additional excitation, due to the nonzero resistivity, is also possible [26]. We have to point out that, although the background quantities depend on time, in the search for a dispersion relation, we treat the perturbations' amplitudes $\left(A_{i} \mathrm{~s}\right)$ as constants (at least initially). In this way, our search for potential waveforms, is not disturbed by the inherent non-linearity introduced by the curved background. Nevertheless, once the potential waveforms are determined, their interaction with the curved space-time in the presence of an external magnetic field, implies that, for $t>0$, the time-dependence of their amplitudes is a priori expected [clf Eq (34), below].

The combination of Eqs (15) and (16) results in the dispersion relation

$$
D_{r}(k, n)+\imath D_{i}(k, n)=0
$$

the real part of which is written in the form

$$
\begin{aligned}
D_{r}(k, n)= & -n^{2}\left[\frac{22-9 \gamma}{9(2-\gamma)}\right] \\
& +k^{2}\left[c_{s}^{2}+\frac{u_{A}^{2}}{2-\gamma}\right]+J_{1}-R_{3}-\frac{u_{A}^{2}}{2-\gamma} R_{1}
\end{aligned}
$$

where, we have set

$$
J_{1}=-(1+\gamma)\left(\frac{16}{9} \theta^{2}+2 \sigma^{2}\right)-\left(1+3 c_{s}^{2}\right)\left(1+c_{s}^{2}\right) \rho
$$

and

$$
\begin{aligned}
& R_{1}=-\frac{1}{6}(33 \gamma+29) \theta^{2}+4 \sigma^{2}+2 \rho(1+2 \gamma)+\frac{10}{3} H^{2}, \\
& R_{3}=\frac{u_{A}^{2}}{9}\left[(18 \gamma-122) \theta^{2}+15(1+\gamma) \rho\right]
\end{aligned}
$$

The vanishing of Eq (18) leads to a critical wave-number, for which the cosmological perturbations under consideration are stable (oscillating); namely, $n^{2} \geq 0$

$$
\begin{aligned}
& k^{2} \geq k_{r_{J}}^{2}(\gamma, t)=\left[-J_{1}+R_{3}+\frac{u_{A}^{2}}{2-\gamma} R_{1}\right]\left[c_{s}^{2}+\frac{u_{A}^{2}}{2-\gamma}\right]^{-1} \\
& \delta u=0
\end{aligned}
$$

As a consequence, for $k^{2}<k_{r_{J}}^{2}$, the small-scale fluctuations of the cosmic-medium become unstable, acquiring an imaginary frequency $\left[n_{k}^{2}(t)<0\right]$. In other words, they are no longer oscillating, but evolve exponentially with time (Jeans-like instability).

In the limiting case where $\gamma=\frac{1}{3}$ (FRW radiation model), we have $H=0=u_{A}^{2}, \sigma^{2}=0, \theta \neq 0$ and $\mathrm{Eq}$ (18) takes the simpler form obtained in [16]. In this case, a Jeans instability arises at wave-numbers $k^{2}<k_{r_{J}}^{2}\left(\frac{1}{3}, t\right)$, where

$$
k_{r_{J}}^{2}\left(\frac{1}{3}, t\right) c_{s}^{2}=\left(1+c_{s}^{2}\right)\left(1+3 c_{s}^{2}\right) \rho+\frac{16}{9}\left(1+c_{s}^{2}\right) \theta^{2}
$$

On the other hand, for $\gamma \rightarrow 1$ (Zel'dovich ultra-stiff matter) we obtain $H=0=u_{A}^{2}$, while $\sigma^{2} \neq 0, \theta \neq 0$ and Eq (18) also yields the corresponding result of [16]; namely,

$$
k_{r_{J}}^{2}(1, t) c_{s}^{2}=\left(1+c_{s}^{2}\right)\left(1+3 c_{s}^{2}\right) \rho+\frac{4}{3}\left(\frac{16}{9} \theta^{2}+2 \sigma^{2}\right)
$$

Finally, in the extreme case of a static model, i.e. $\theta=0$ and $\sigma=0$, we recover the well-known Jackson's solution [36]. Accordingly, we conclude that, the critical wave-number (21) possesses the correct asymptotic behavior in every limiting case.

The rhs of Eq (21) is a function of time, as well as of $\gamma$. In particular,

$$
k_{r_{J}}^{2}(\gamma, t)=\frac{P_{5}(\gamma) / Q_{3}(\gamma)}{(\gamma+1)^{2} t^{2}}
$$

where, for all $\gamma$ in the range $\left[\frac{1}{3}, 1\right)$,

$$
\begin{aligned}
Q_{3}(\gamma) & =36 \gamma(3-\gamma)(2-\gamma) \\
& +18(1-\gamma)(3 \gamma-1)>0
\end{aligned}
$$

and

$$
\begin{aligned}
P_{5}(\gamma) & =2(1+\gamma)(3-\gamma)(2-\gamma)\left(27 \gamma^{2}+36 \gamma+161\right) \\
& +(1-\gamma)(3 \gamma-1)(2-\gamma)\left(-15 \gamma^{2}+174 \gamma-931\right) \\
& +3(1-\gamma)(3 \gamma-1)\left(9 \gamma^{2}-106 \gamma-99\right)>0
\end{aligned}
$$

i.e. they are (positive) polynomials of $\gamma$, of the thirdand the fifth-order, respectively (see Fig. 2). We observe that, for every $\frac{1}{3} \leq \gamma<1$, we have

$$
k_{r_{J}}^{2}(\gamma, t) \leq k_{r_{J}}^{2}\left(\frac{1}{3}, t\right)
$$

and therefore

$$
\lambda_{r_{J}}(\gamma, t) \geq \lambda_{r_{J}}\left(\frac{1}{3}, t\right)
$$


In other words, the more we depart from a FRW radiation model, the larger is the scale at which gravitomagnetic instabilities can become prominent. Departure from $\gamma=\frac{1}{3}$, suggests that the initial fortification of the magnetic-field strength - for $\frac{1}{3} \leq \gamma \leq 0.6$ - is compensated by a subsequent decrease - for $0.6<\gamma<1$ (in connection see Fig. 1). However, in any case, the corresponding length-scale grows - at least up to $\gamma \lesssim 0.85$, after which it remains practically constant (Fig. 2). Therefore, we may conclude that, any non-vanishing ambient magnetic field disfavors small-scale (sub-horizonal) condensations within a non-resistive plasma-fluid. This result is compatible to what has already been found in the isotropic case (e.g. see [13], [14]).

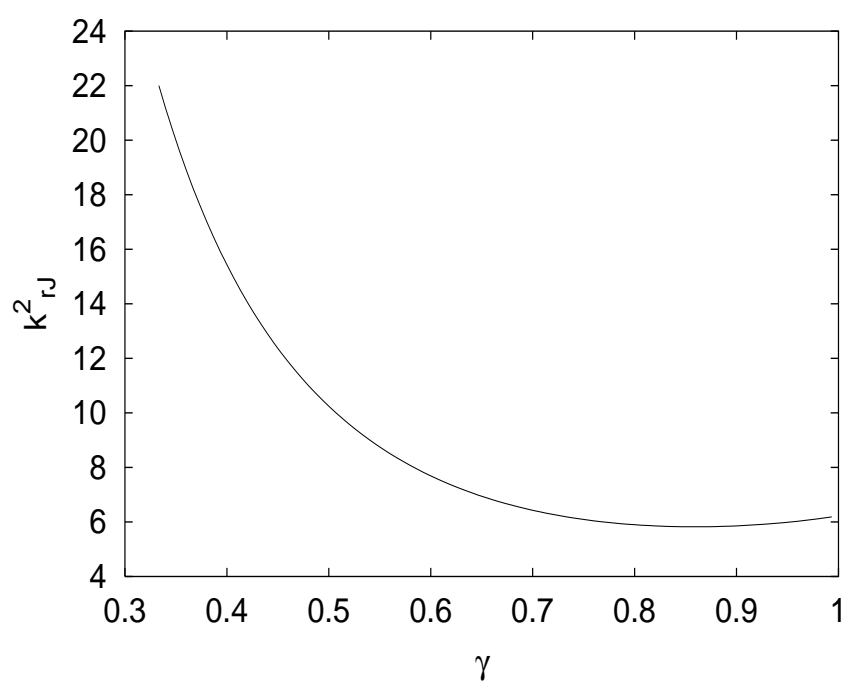

FIG. 2: The critical wave-number $k_{r_{J}}^{2}$ (in cgs units) at a fixed time $\left(t \simeq 10^{-21} \mathrm{sec}\right)$, as a function of $\gamma$ taking values in the range $\left[\frac{1}{3}, 1\right)$.

Since $k_{r_{J}} \sim t^{-1}$, the entirety of critical wave-numbers in the form (24) vanish only at $t \rightarrow \infty$. Hence, in the curved space-time (5) we may always find a lengthscale involving a gravito-magnetic instability. However, the MHD perturbations considered, correspond to lowfrequency waves and, hence, these results should be valid (mainly) at the late stages of the radiation epoch (probably around recombination). For every $t$ within the radiation era, we may use Eqs (24) - (26) to estimate the corresponding critical length-scales (see also Fig. 2). In accordance,

$$
k_{r_{J}} \simeq(2.5-4.7) \times \frac{1}{t}
$$

(in geometrical units) where, the higher values correspond to $\gamma$ in the vicinity of $\frac{1}{3}$ (in particular, $\gamma \lesssim 0.5$ ), while, the lower ones to the rest of the range. The corresponding Jeans length reads

$$
\lambda_{r_{J}}=\frac{2 \pi}{k_{r_{J}}} \simeq(1.3-2.5) \times t
$$

in geometrical units or

$$
\lambda_{r_{J}} \simeq(4-7.5) \times 10^{10} \times t
$$

in $c g s$ units. It is easy to verify that, for all $t$ within the radiation era, these scales are comparable to the horizon size along the $x y$-plane $\left(\ell_{H \perp}=2 c t=6 \times 10^{10} \times t\right.$, in $\mathrm{cgs}$ units). In fact,

$$
0.67 \ell_{H \perp} \leq \lambda_{r_{J}} \leq 1.25 \ell_{H \perp}
$$

with the lower values (i.e. those for which $\lambda_{r_{J}} \lesssim \ell_{H \perp}$ ) corresponding to $\gamma \lesssim 0.5$ and the higher ones to $0.5<$ $\gamma<1$. Confining ourselves to sub-horizonal scales (e.g. see [37]), we may conclude that, as regards favorism of gravito-magnetic instabilities in a cosmological model of the form (5), the values of $\gamma>0.5$ should be discarded.

As regards the sub-horizonal perturbations, for a fixed $k$, Eqs (21) and/or (24) introduce to the evolution of the corresponding mode a critical time-scale $\left(t_{k_{r_{J}}}\right)$ in the following sense: As long as

$$
t<\frac{\sqrt{P_{5}(\gamma) / Q_{3}(\gamma)}}{(\gamma+1) k}=t_{k_{r_{J}}}
$$

the cosmological perturbation involved is unstable, i.e. $n_{k}^{2}\left(t<t_{k_{r_{J}}}\right)<0$ and grows exponentially with time [38]

$$
\begin{aligned}
& \delta \rho(t), \delta H(t)=A_{i} \exp \left(-\imath \int^{t} n_{k}\left(t^{\prime}\right) d t^{\prime}\right) \\
= & A_{i}\left[\frac{\sqrt{P_{5}}-\sqrt{P_{5}-(\gamma+1)^{2} Q_{3} k^{2} t^{2}}}{\sqrt{P_{5}}+\sqrt{P_{5}-(\gamma+1)^{2} Q_{3} k^{2} t^{2}}}\right] \\
\times & \exp \left[-a(\gamma) \sqrt{P_{5}-(\gamma+1)^{2} Q_{3} k^{2} t^{2}}\right]
\end{aligned}
$$

where $A_{i}$ stands either for $A_{\rho}$ or $A_{H}$,

$$
a(\gamma)=\frac{1}{2(\gamma+1) \sqrt{(22-9 \gamma)(3-\gamma)}}
$$

and the exponentially decaying solutions - those with the plus sign in the exponents - are neglected. With the aid of $\mathrm{Eq}(24), \mathrm{Eq}(34)$ is written in the form

$$
\begin{aligned}
\delta \rho(k), \delta H(k) & =A_{i}\left[\frac{1-\sqrt{1-\left(\frac{k}{k_{r_{J}}}\right)^{2}}}{1+\sqrt{1-\left(\frac{k}{k_{r_{J}}}\right)^{2}}}\right] \\
& \times e^{-a(\gamma) \sqrt{P_{5}(\gamma)} \sqrt{1-\left(\frac{k}{k_{r_{J}}}\right)^{2}}}
\end{aligned}
$$

In the limit $k \rightarrow k_{r_{J}}$, Eq (36) yields $\delta \rho, \delta H=A_{i}$ (the constant amplitude of an oscillating perturbation), while, for $k \ll k_{r_{J}}$ we obtain

$$
\delta \rho(k), \delta H(k) \gtrsim A_{i}\left(\frac{k_{r_{J}}}{k}\right)^{a(\gamma) \sqrt{P_{5}(\gamma)}}>A_{i}
$$

Accordingly, condensations of the corresponding lengthscale $\left(\lambda_{r_{J}}\right)$ will collapse in Jeans' fashion, while, at the 
same time, the fluctuations of the magnetic field will grow, resulting in a gravito-magnetic instability, as well as in the amplification of the background magnetic field (in connection, see [25], [39]).

The behavior of the (large scale) magnetized perturbations in the anisotropic model under consideration, is completely different than their behavior in the isotropic FRW model. In that case, both the Newtonian [11] and the GR study [13], [14] have verified a negative role of the magnetic field in the aggregation of matter on large scales. In particular, it has been shown that, on scales between the Jeans length and the horizon, the density perturbations may undergo a power-law evolution similar to that of the non-magnetized case (e.g. see [1]), but their growth-rate is reduced by an amount proportional to the magnetic field's strength. Therefore, in the isotropic case, the external field disfavors viable matter condensations.

As regards the anisotropic case, the density perturbation at any spatial location can be obtained by a superposition of modes with different wave-numbers

$$
\delta \rho(\vec{x}, t)=\int \frac{d^{3} k}{(2 \pi)^{3}} \delta \rho(k) e^{\imath \vec{k} \cdot \vec{x}}
$$

The strength of such a perturbation can be measured by the value of $|\delta \rho(\vec{x}, t)|^{2}$. From Eq (38), it follows that modes in the range $(k, k+d k)$ contribute to $|\delta \rho(\vec{x}, t)|^{2}$ an amount proportional to $|\delta \rho(k)|^{2} d^{3} k$. Writing the contribution as

$$
|\delta \rho(k)|^{2} d^{3} k=4 \pi|\delta \rho(k)|^{2} k^{2} d k=4 \pi k^{3}|\delta \rho(k)|^{2} d(\ln k)
$$

we see that each logarithmic $k$-interval contributes to $|\delta \rho(\vec{x}, t)|^{2}$ an amount $\mathcal{P}_{k}^{2} \sim k^{3}|\delta \rho(k)|^{2}$, which represents the so-called power spectrum [1]. With the aid of Eq (37), the power spectrum of the MHD perturbations under consideration is written in the form

$$
\mathcal{P}_{k}^{2} \sim k^{3-2 a(\gamma)} \sqrt{P_{5}(\gamma)}
$$

which, for $\gamma=\frac{1}{3}$ reads $\mathcal{P}_{k} \sim k^{-0.9}$, while, in the limiting case $\gamma \rightarrow 1$, it results in $\mathcal{P}_{k} \sim k^{-0.6}$. Notice that, although both cases correspond to the absence of the ambient magnetic field, the spectral indices involved are different. This difference arises due to the stiffness of the Universe matter-content as $\gamma \rightarrow 1\left(c_{s} \rightarrow c\right)$. The explicit form of the spectral index

$$
\nu(\gamma)=\frac{k}{\delta \rho} \frac{d}{d k} \delta \rho=\frac{3}{2}-a(\gamma) \sqrt{P_{5}(\gamma)}
$$

as a function of $\gamma \in\left[\frac{1}{3}, 1\right)$, is presented in Fig. 3. We note that, for every $\gamma$ in this range, we have $\nu(\gamma)<0$. Once again, we verify that most of the power is concentrated on large scales (small wave-numbers).

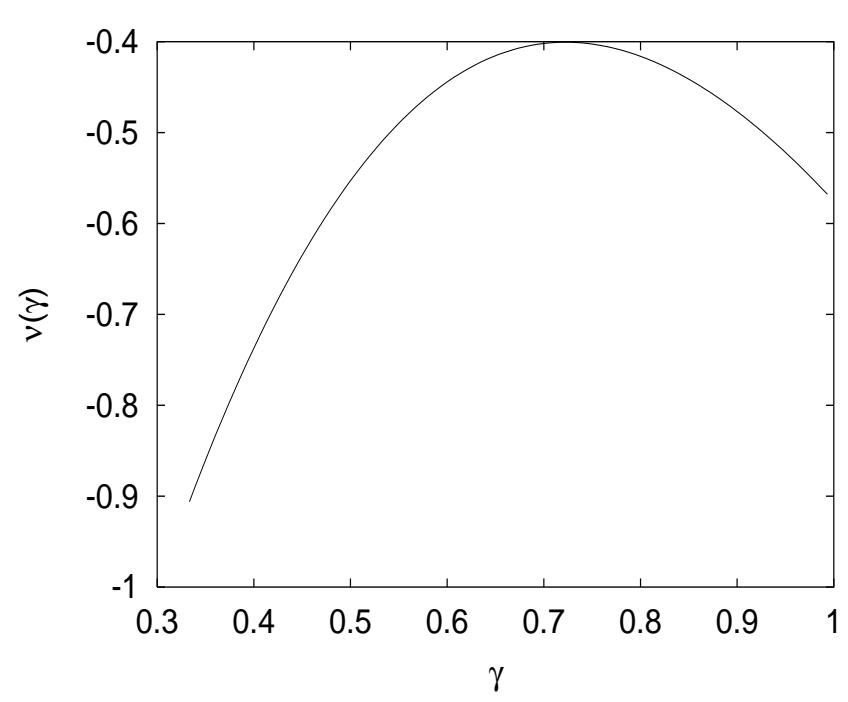

FIG. 3: The spectral index $\nu(\gamma)$ of the unstable perturbations as a function of $\gamma$, taking values in the range $\left[\frac{1}{3}, 1\right)$.

\section{JEANS LENGTHS IN A RESISTIVE PLASMA}

It has been argued [26] that, prior to recombination, the conductivity of the Universal plasma fluid is finite, although it may reach at very high values, of the order $\left.10^{12}-10^{13} \mathrm{~m} / \mathrm{ohm}\right)$. Accordingly, the study of MHD perturbations in a Bianchi Type I model has indicated that the finite conductivity (non-zero resistivity) could result in an exponential growth of the density perturbations. This argument was accordingly confirmed in the Newtonian limit [11], but not in the relativistic one. In this Section, we shall attempt to re-examine the above statements in the relativistic limit. The equations which govern the hydrodynamics of a magnetized, resistive fluid in the frame of GR, are identical to Eqs (1) and (2) being supplemented by the Maxwell equations

$$
\dot{H}^{\alpha}=\left(\sigma_{\beta}^{\alpha}+\omega_{\beta}^{\alpha}\right) H^{\beta}-\frac{2}{3} \theta H^{\alpha}+u^{\alpha} H_{; \gamma}^{\gamma}+\eta h^{\gamma \beta} H_{; \gamma \beta}^{\alpha}
$$

which coincide to Eqs (3), with the addition of an extra term due to the resistivity $(\eta)$ of the cosmic medium.

In this case, as regards the propagation of cosmological perturbations along the $\hat{x}$-direction, Eq (16) reads

$$
(H \delta H)\left[(2-\gamma) \theta+k^{2} \eta+\imath n\right]=\frac{u_{A}^{2}}{1+\gamma}[\theta(1+\gamma)-\imath n] \delta \rho
$$

Accordingly, the vanishing of the real part of the dispersion relation results in

$$
\begin{aligned}
& n^{2}\left[\frac{22-9 \gamma}{9} \theta+k^{2} \eta\right]=(2-\gamma)\left\{k^{4} \frac{c_{s}^{2} \eta}{2-\gamma}\right. \\
+ & k^{2}\left[\left(c_{s}^{2}+\frac{u_{A}^{2}}{2-\gamma}\right) \theta+\frac{\eta}{2-\gamma}\left(J_{1}-R_{3}\right)\right] \\
+ & \left.\left(J_{1}-R_{3}-\frac{u_{A}^{2}}{2-\gamma} R_{1}\right) \theta\right\}, \quad \delta u=0
\end{aligned}
$$


Eq (43) can be cast in the form

$$
n^{2}\left[\frac{22-9 \gamma}{9} \theta+k^{2} \eta\right]=k^{4} m_{4}+k^{2} m_{2}+m_{0}
$$

where, for every $\gamma \in\left[\frac{1}{3}, 1\right)$,

$$
\begin{aligned}
m_{4} & =c_{s}^{2} \eta>0 \\
m_{0} & =(2-\gamma) \theta\left(J_{1}-R_{3}\right)-\theta u_{A}^{2} R_{1} \\
& =-\frac{P_{5}(\gamma)}{18(3-\gamma)(1+\gamma)^{3} t^{3}}<0
\end{aligned}
$$

and

$$
\begin{aligned}
m_{2} & =c_{s}^{2}(2-\gamma) \theta+\theta u_{A}^{2}+\left(J_{1}-R_{3}\right) \eta \\
& =\frac{Q_{3}(\gamma)}{18(3-\gamma)(1+\gamma) t}-\eta \frac{B_{4}(\gamma)}{36(3-\gamma)(1+\gamma)^{2} t^{2}}
\end{aligned}
$$

while

$$
\begin{aligned}
B_{4}(\gamma) & =2(1+\gamma)(3-\gamma)\left(27 \gamma^{2}+36 \gamma+161\right) \\
& +(1-\gamma)(3 \gamma-1)\left(-15 \gamma^{2}+174 \gamma-931\right)>0
\end{aligned}
$$

The allowed wave-numbers for stable (oscillating) perturbations can be easily obtained by Eq (44), admitting that $n^{2} \geq 0$. Accordingly,

$$
m_{4} k^{4}+m_{2} k^{2}+m_{0} \geq 0
$$

Since $m_{2}^{2}-4 m_{4} m_{0}>0$, the trinomial on the lhs of Eq (48) possesses the couple of real roots

$$
k_{r_{J(1,2)}}^{2}=\frac{-m_{2}}{2 m_{4}} \pm \frac{1}{2 m_{4}} \sqrt{m_{2}^{2}-4 m_{4} m_{0}}
$$

For small values of $\eta(\eta \rightarrow 0)$, i.e. within the context of an ideal-plasma cosmology, the corresponding wavenumbers behave as

$$
\begin{aligned}
k_{r_{(1,2)}}^{2} \simeq & -\frac{1}{36 \eta \gamma} \frac{Q_{3}(\gamma)}{(3-\gamma)(1+\gamma) t} \\
& \pm \frac{1}{36 \eta \gamma} \frac{Q_{3}(\gamma)}{(3-\gamma)(1+\gamma) t} \\
& \pm \frac{P_{5}(\gamma) / Q_{3}(\gamma)}{(1+\gamma)^{2} t^{2}}
\end{aligned}
$$

and therefore, now, the condition for unstable perturbations reads

$$
\begin{aligned}
k_{r_{J_{1}}}^{2} & \leq k^{2} \leq k_{r_{J_{2}}}^{2} \Rightarrow \\
0 & <k^{2} \leq k_{r_{J_{2}}}^{2}=\frac{P_{5}(\gamma) / Q_{3}(\gamma)}{(1+\gamma)^{2} t^{2}}
\end{aligned}
$$

i.e. it is identical to the corresponding non-resistive one.

On the other hand, in the limit of large $\eta$, we obtain

$$
\begin{aligned}
k_{r_{J_{(1,2)}}}^{2} & =\frac{B_{4}(\gamma)}{72 \gamma(3-\gamma)(1+\gamma)^{2} t^{2}} \\
& \pm \frac{B_{4}(\gamma)}{72 \gamma(3-\gamma)(1+\gamma)^{2} t^{2}}+O\left(\frac{1}{\eta}\right)
\end{aligned}
$$

In accordance, as long as

$$
0<k^{2} \leq k_{r_{J_{2}}}^{2}=\frac{B_{4}(\gamma)}{36 \gamma(3-\gamma)(1+\gamma)^{2} t^{2}}
$$

the condition (48) is not fulfilled and the corresponding cosmological fluctuations are unstable and grow exponentially with time $\left(n_{k}^{2}<0\right)$. As a consequence, condensations of the corresponding length-scale will collapse in the Jeans' fashion, revealing an indirect resistive instability [the rhs of Eq (53) is independent of $\eta$ ].

In what follows, we compare the length-scales arising by the critical wave-numbers of the resistive and the nonresistive case. Our aim is to examine the contribution of resistivity in achieving more realistic (sub-horizonal) scales of gravito-magnetic instability, than those predicted by the non-resistive fluid. To do so, we consider the ratio of the critical wave-numbers (53) and (24), which is written in the form

$$
\frac{k_{r e s}^{2}}{k_{n o n}^{2}}=\frac{B_{4}(\gamma) Q_{3}(\gamma)}{36 \gamma(3-\gamma) P_{5}(\gamma)}
$$

and is depicted in Fig. 4. First of all, we observe that for $\gamma=\frac{1}{3}$ and/or $\gamma=1$, i.e. in the absence of the ambient magnetic field, we obtain $k_{r e s}=k_{n o n}$. This not an unexpected result, since $\eta$ is introduced to the MHD analysis through the Maxwell equation (41), which involves a non-vanishing magnetic-field strength. On the other hand, a large resistivity can modify quite significantly the scales involved in a gravito-magnetic instability and especially those around the value $\gamma=\frac{2}{3}$ (the peak in Fig. 4), where the non-resistive analysis predicts a superhorizonal Jeans length $\left[\lambda_{\text {non }}\left(\gamma=\frac{2}{3}, t\right)=1.20 \ell_{H \perp}(t)\right]$. Accordingly, for $\gamma=\frac{2}{3}$, we obtain

$$
k_{\text {res }} \simeq 1.2 k_{\text {non }} \Rightarrow \lambda_{\text {res }} \simeq 0.83 \lambda_{\text {non }} \lesssim \ell_{H \perp}(t)
$$

Therefore, we may conclude that, as compared to the ideal-plasma case, gravito-magnetic instabilities within a magnetized-fluid are particularly favored by a large resistivity, in the sense that it prolongs the range of the potential values of $\gamma$ (and therefore the number of the corresponding cosmological models) which admit subhorizonal scales of such an instability (from $\frac{1}{3} \leq \gamma \lesssim \frac{1}{2}$ in the non-resistive case, to $\frac{1}{3} \leq \gamma \lesssim \frac{2}{3}$ in the fully-resistive one).

This result, which is based on the fact that the critical (Jeans) wave-number in the resistive case is always larger than the corresponding non-resistive one (clf Fig. 4), has a clear physical interpretation: Indeed, the invariant form of Ohm's law for a locally neutral plasma is written as [35]

$$
J^{\mu}=\frac{1}{\eta} F^{\mu \nu} u_{\nu}
$$

where, $J^{\mu}$ is the current density and $F^{\mu \nu}$ is the Faraday tensor. According to it, besides the convective field, a non-zero resistivity favors also convective currents. As a 


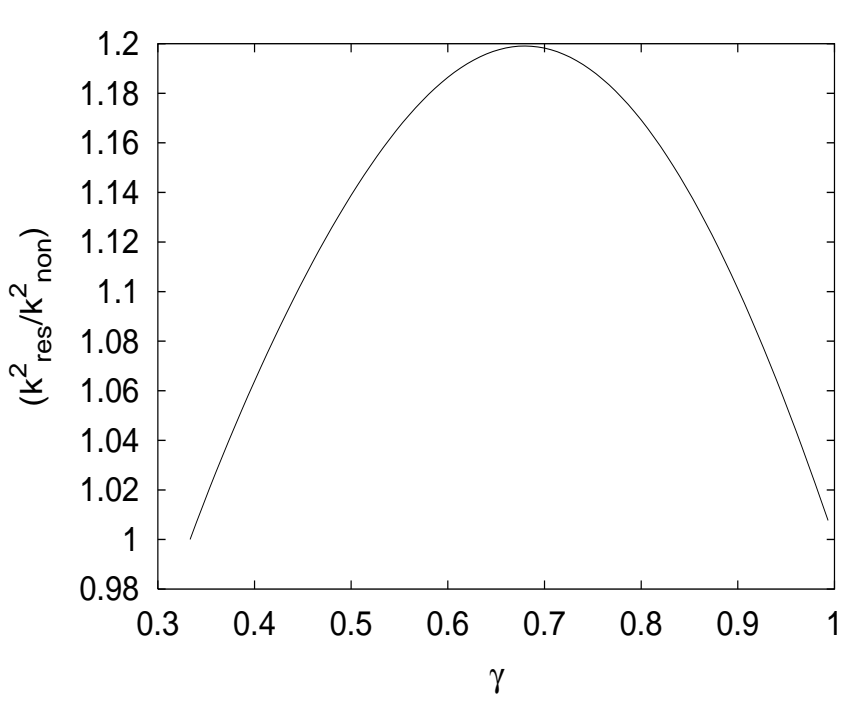

FIG. 4: The ratio $k_{\text {res }}^{2} / k_{\text {non }}^{2}$ of the unstable perturbations as a function of $\gamma$, taking values in the range $\left[\frac{1}{3}, 1\right)$.

consequence, for $\eta \neq 0$, the energy available to be absorbed by the perturbation quantities is larger than that of the non-resistive case. This energy-excess will lead to the increase of the perturbations' frequency and hence to the amplification of their wave-number, as well (see also $[25])$.

\section{CONCLUSIONS}

Gravitational instabilities in a magnetized FRW Universe, in which the magnetic field was assumed to be too weak to destroy the isotropy of the model, are known and have been studied in the past [13], [14], [16]. However, mathematically speaking, the spatial isotropy of the FRW Universe is not compatible with the presence of large-scale magnetic fields. In fact, an anisotropic cosmological model can and should be imposed for the treatment of magnetic fields whose coherent length is comparable to the horizon length [27]. Interesting homogeneous and anisotropic cosmological models of Bianchi Type I with an ambient magnetic field are known [28] and have been used to discuss astrophysical implications [6], [19], [25], [29], [31].

In this article, we have used the GR version of the MHD equations [18] in a homogeneous and anisotropically expanding, self-gravitating, magnetized plasma, to study the gravito-magnetic excitation of cosmological perturbations. Accordingly, we have found that:

- In a non-resistive medium:

1. In contrast to the isotropic case, where the role of the magnetic field (as an agent opposing the perturbations' growth) is clear, there is a wide class of anisotropic, magnetized cosmological models - parameterized by $\gamma$ in the range $\frac{1}{3} \leq \gamma \lesssim 0.5$, for which a gravitomagnetic instability can lead to sub-horizonal magnetized condensations [clf Eqs (29), (31) and (32)].

2. We have determined explicitly the power spectrum of the unstable cosmological perturbations [Eq (37)], which indicates that most of the power is concentrated on large scales, close to the horizon [clf $\mathrm{Eq}(40)]$.

- In a resistive medium:

1. The critical wave-numbers so obtained, exhibit a delicate dependence on resistivity $[\mathrm{Eq}$ (52)], resulting in the reduction of the corresponding Jeans lengths to smaller scales (well below the horizon) than the non-resistive ones. In fact, the non-zero resistivity induces convective currents, resulting in the enhancement of the perturbations' energy (frequency) and hence in the reduction of their comoving wavelength.

2. Furthermore, the non-zero resistivity prolongs the range of the potential values of $\gamma$ (and therefore the number of the corresponding cosmological models) which admit sub-horizonal scales of such an instability (from $\frac{1}{3} \leq \gamma \lesssim \frac{1}{2}$ in the non-resistive case, to $\frac{1}{3} \leq \gamma \lesssim \frac{2}{3}$ in the fully-resistive one) [clf Eq (54)].

\section{Acknowledgments}

The authors would like to thank Dr Heinz Ishliker and Dr Christos G. Tsagas for several helpful discussions. We also thank the anonymous referee for his critical comments and his useful suggestions, which greatly improved the article's final form. Finally, financial support from the Greek Ministry of Education under the Pythagoras programm, is also gratefully acknowledged.

\section{Appendix A}

The vanishing of the imaginary part, $D_{i}(k, n)$, of the dispersion relation (17) results in

$$
n^{2}\left[1+\frac{u_{A}^{2}}{1+\gamma}\right]=k^{2}\left[c_{s}^{2}-\frac{u_{A}^{2}}{1+\gamma}\right]+\frac{4}{9}\left(\theta-R_{4}\right)(2-\gamma) \theta+
$$

$$
+J_{1}-R_{3}+\frac{u_{A}^{2}}{1+\gamma}\left[R_{1}+\theta R_{2}(1+\gamma)\right]
$$

where

$$
R_{2}=\frac{1}{2} \theta(3 \gamma+1)
$$


and

$$
R_{4}=-\frac{u_{A}^{2}}{2(1+\gamma)} \theta(18 \gamma-122)
$$

In this case, the requirement $n^{2} \geq 0$ for oscillating (stable) fluctuations leads to the critical (Jeans) wavenumber

$$
\begin{gathered}
k_{i_{J}}^{2}\left[c_{s}^{2}-\frac{u_{A}^{2}}{1+\gamma}\right]=-\frac{4}{9}\left(\theta-R_{4}\right)(2-\gamma) \theta- \\
-J_{1}+R_{3}-\frac{u_{A}^{2}}{1+\gamma}\left[R_{1}+(1+\gamma) \theta R_{2}\right]
\end{gathered}
$$

Once again, for every $\gamma \in\left[\frac{1}{3}, 1\right)$ the rhs of Eq (A4) is of the form (24), thus remaining positive for all $t$. Therefore, in a non-resistive fluid, the small-scale cosmological perturbations with $k<k_{i_{J}}$ are unstable and grow exponentially with time. Hence, formations of the corresponding length-scale will collapse as a result of a gravito-magnetic instability.

On the other hand, as regards the propagation of MHD fluctuations in a resistive fluid, the vanishing of the imaginary part of the corresponding dispersion relation yields

$$
\begin{gathered}
n^{2}\left[1+\frac{u_{A}^{2}}{1+\gamma}\right]=k^{2}\left[c_{s}^{2}-\frac{u_{A}^{2}}{1+\gamma}+\eta \frac{4}{9}\left(\theta-R_{4}\right)\right]+ \\
+J_{1}-R_{3}+\frac{4}{9}\left(\theta-R_{4}\right)(2-\gamma) \theta+ \\
+\frac{u_{A}^{2}}{1+\gamma}\left[R_{1}+R_{2} \theta(1+\gamma)\right]
\end{gathered}
$$

Accordingly, for $n^{2} \geq 0$ we obtain $k^{2} \geq k_{i_{J}}^{2}$, where

$$
k_{i_{J}}^{2}=\left[c_{s}^{2}-\frac{u_{A}^{2}}{1+\gamma}+\eta \frac{4}{9}\left(\theta-R_{4}\right)\right]^{-1} \times
$$

$$
\times\left\{-J_{1}+R_{3}-\frac{4}{9}\left(\theta-R_{4}\right)(2-\gamma) \theta-\right.
$$

$$
\left.-\frac{u_{A}^{2}}{1+\gamma}\left[R_{1}+R_{2} \theta(1+\gamma)\right]\right\}
$$

In this case, the critical wave-numbers so obtained $\left(k_{i_{J}}^{2}\right)$, are weakly-dependent on resistivity. In particular, around recombination the resistivity is related to the background temperature (T) according to [11], [40], [41]

$$
\eta=\eta_{n} T^{-3 / 2}
$$

where $\eta$ (in geometrical units) is measured in sec. At the late stages of the radiation epoch $T=2.7(1+z)$, where $z$ is the cosmological redshift, while $\eta_{n}=7 \times 10^{10}$ for a weakly ionized gas and $\eta_{n}=10^{13}$ for the fully ionized one. Therefore, at $z_{r e c} \simeq 1500$, the product $\eta \times \theta$ (in geometrical units) is dimensionless, admitting the value $\eta \times \theta \sim 2 \times \frac{10^{-8}}{(1+\gamma)}$ (for a weakly ionized gas). For this reason, the influence of resistivity in $\mathrm{Eq}(\mathrm{A} 6)$, as compared to Eq (A4), is of minor interest, since it simply adds a term of the order $10^{-8}$ to the evaluation of $k_{i_{J}}^{2}$.
[1] Padmanabhan T, 1993, Structure Formation in the Universe, Cambridge University Press, Cambridge

[2] Mukhanov V F, 2005, Physical Foundations of Cosmology, Cambridge University Press, Cambridge

[3] Jeans J, 1902, Phil Trans R Soc 25, 895

[4] Jeans J, 1928, Astronomy and Cosmology (2nd Edition) Cambridge University Press, Cambridge (reprinted by Dover Publications Inc, 1961), pp 345 - 350

[5] Weinberg S, 1972, Gravitation and Cosmology, Wiley and Sons, New York

[6] Zel'dovich Ya B \& Novikov I D, 1983, Relativistic Astrophysics - Volume 2: The Structure and the Evolution of the Universe, Chicago University Press, Chicago, pp 501 - 506

[7] Lifshitz E M, 1946, J Phys (USSR), 10, 116

[8] Weinberg S, 1971, ApJ 168, 175

[9] Eckart C, 1940, Phys Rev 58, 29
[10] Landau L D \& Lifshitz E M, 1959, Fluid Mechanics, Pergamon Press, London

[11] Hacyan S, 1983, ApJ 273, 421

[12] Ruzmaikina T V \& Ruzmaikin A A, 1971, Sov Astron 14, 963

[13] Tsagas C G \& Barrow J D, 1997, Class Quantum Grav 14, 2539

[14] Tsagas C G \& Barrow J D, 1998, Class Quantum Grav 15, 3523

[15] Durrer R, Kahniashvil T \& Yates A, 1998, Phys Rev D 58, 123004

[16] Papadopoulos D B, Vlahos L \& Esposito F P, 2001, A\&A 382, 1

[17] Wasserman I, 1978, ApJ 224, 337

[18] Papadopoulos D \& Esposito FP, 1982, ApJ 257, 10

[19] Zel'dovich Ya B, Ruzmaikin A A \& Sokoloff D D, 1983, Magnetic Fields in Astrophysics, Gordon and Breach, 
New York

[20] Adams J, Danielsson U H \& Rubinstein H, 1996, Phys Lett B 388, 253

[21] Barrow J D, Ferreira P G \& Silk J, 1997, Phys Rev Lett $\mathbf{7 8}, 3610$

[22] Jedamzik K, Katalinic V \& Olinto A, 1998 Phys Rev D $\mathbf{5 7}, 3264$

[23] Jedamzik K, Katalinic V \& Olinto A, 2000, Phys Rev Lett 85, 700

[24] Barrow J D, Maartens R \& Tsagas C G, 2006, "Cosmology with inhomogeneous magnetic fields", arXiv: astro$\mathrm{ph} / 0611537$

[25] Kuiroukidis A, Kleidis K, Papadopoulos D B \& Vlahos L, 2007, A\&A 471, 409

[26] Fennelly A J, 1980, Phys Rev Lett 44, 955

[27] Tsagas C G \& Maartens R, 2000, Class Quantum Grav $\mathbf{1 7}, 2215$

[28] Thorne K S, 1967, ApJ 148, 51

[29] Jacobs K C, 1969, ApJ 155, 379

[30] Raychaudhuri A, 1955, Phys Rev 98, 1123

[31] Jacobs K C, 1968, ApJ 153, 661
[32] Cowling T G, 1941, MNRAS 101, 367

[33] Zel'dovich Ya B, 1979, Creation of particles by the gravitational field in Demianski M (ed) Physics of the Expanding Universe, Springer Verlag, New York

[34] Birrell N D \& Davies P C W, 1982, Quantum Fields in Curved Space, Cambridge University Press, Cambridge

[35] Krall N A and Trivielpiece A W, 1973 Principles of Plasma Physics, Mc Graw Hill, New York

[36] Jackson J C, 1972, Proc R Soc Lond A 328, 561

[37] Mukhanov V F, 2005, Physical Foundations of Cosmology, Cambridge University Press, Cambridge

[38] Gradshteyn I S \& Ryzhik I M, 1965, Table of Integrals, Series and Products, Academic Press, New York, Eq 2.275 , p. 87

[39] Kleidis K, Kuiroukidis A, Papadopoulos D B \& Vlahos L, 2008, IJMP A 23, 1697

[40] Lang K R, 1974, Astrophysical Formulae, SpringerVerlag, New York, p. 227

[41] Peebles P J E, 1980, The Large-Scale Structure of the Universe, Princeton University Press, Princeton NJ, p. 397 